\title{
Pyogenic liver abscess and the emergence of Klebsiella as an etiology: a retrospective study
}

This article was published in the following Dove Press journal:

International Journal of General Medicine

23 December 2013

Number of times this article has been viewed

\section{Ahmad H Ali \\ Roger D Smalligan \\ Mashrafi Ahmed \\ Faisal A Khasawneh \\ Department of Internal Medicine, Texas Tech University Health Sciences Center, Amarillo, TX, USA}

Objectives: Pyogenic liver abscess (PLA) is a significant, though uncommon, cause of morbidity in the United States. Recently, Klebsiella has emerged as an important cause of PLA. We analyzed the clinical course, microbiology, and treatment outcomes of patients discharged with PLA. In addition, we sought to examine the incidence of and risk factors for Klebsiella liver abscess (KLA).

Methods: We reviewed the charts of patients who discharged with PLA from two teaching hospitals in West Texas between January 1, 2007 and December 31, 2011.

Results: We identified 49 cases of PLA. Abscess cultures were positive in 23 (48\%) patients. The mean age of the cohort was 56 years (range: $20-83$ years). Sixty percent were male. The most frequent conditions associated with PLA were intra-abdominal infections (ten cases; $20 \%$ ), diabetes mellitus (nine cases; 18\%) and malignancy (nine cases; 18\%). Klebsiella was the most commonly isolated species from the abscess cultures (seven cases; $30 \%$ of all positive abscess cultures). We used univariate and logistic regression analyses to identify the risk factors for KLA. Controlling for age, only malignancy was identified in our cohort as a risk factor for a Klebsiella liver abscess. The overall mortality was $2 \%$.

Conclusion: Klebsiella is emerging as an important cause of liver abscesses. Malignancy may be an important risk factor for Klebsiella liver abscess.

Keywords: pyogenic liver abscess, Klebsiella

\section{Introduction}

Pyogenic liver abscess (PLA), a suppurative infection of the hepatic parenchyma, can develop as a complication of abdominal and biliary infections, or following hematogenous bacterial seeding of the liver. It is often polymicrobial, with Streptococci and Escherichia coli being the most commonly reported pathogens. ${ }^{1,2}$ Klebsiella spp. have emerged in recent years as important causes of monomicrobial liver abscesses, initially reported in a case series in Asia, and subsequently in other countries. ${ }^{3}$ The full spectrum of Klebsiella liver abscesses (KLA), their pathogenesis, associated risk factors, and typical outcomes are still under investigation. ${ }^{4}$

This retrospective study was designed to analyze the clinical features and treatment outcomes of patients with PLA in two teaching hospitals in West Texas. The surprising finding of several KLAs in our population allowed for further characterization of the incidence and risk factors of this condition, which were previously reported primarily among Asian patients. ${ }^{3}$
Correspondence: Ahmad H Ali

Department of Internal Medicine,

Texas Tech University Health Sciences

Center, I 400 S Coulter, Amarillo,

TX, 79106 USA

Tel +l 8063545480

Fax + I 8063545764

Email ahmad.ali@ttuhsc.edu 


\section{Methods}

The medical records of patients admitted to the Northwest Texas Hospital and Baptist St Anthony's Hospital between January 1, 2007 and December 31, 2011 were queried for a discharge diagnosis of PLA, as per the International Classification of Diseases, Ninth Revision, code 572.0. ${ }^{4}$ The facilities are the teaching hospitals for Texas Tech University Health Sciences Center in Amarillo, TX, USA. The study was approved by the Texas Tech University Health Sciences Center's Institutional Review Board in Amarillo, TX, USA (Institutional Review Board number A12-3695).

\section{Definitions}

Cases were included if a patient with PLA was diagnosed by aspirating purulent material from a liver lesion in the presence of symptoms and signs consistent with the condition, laboratory findings, and compatible imaging studies (computed tomography [CT], magnetic resonance imaging, or ultrasonography). Acute respiratory failure was defined as arterial oxygen partial pressure $\left(\mathrm{PaO}_{2}\right) \leq 55 \mathrm{mmHg}$, and/or arterial carbon dioxide partial pressure $\left(\mathrm{PaCO}_{2}\right) \geq 50 \mathrm{mmHg}$, requiring supplemental oxygen therapy or positive pressure ventilation. Septic shock was defined as sepsis-induced hypotension not responsive to adequate volume resuscitation requiring vasopressor therapy.

\section{Data collection}

The charts of patients with PLA were reviewed and the following data were collected:

1. Demographics: age, sex and, if available, ethnicity.

2. Comorbid conditions already reported to be associated with PLA: diabetes mellitus; malignancy; intra-abdominal infection(s); and hepatobiliary pathology.

3. The presence of other comorbid conditions: congestive heart failure; chronic kidney disease; and liver cirrhosis.

4. Presenting clinical features: fever; chills; abdominal pain; nausea; vomiting; diarrhea; acute respiratory failure; acute kidney injury; and septic shock.

5. Admission laboratory values including: white blood cell count; hemoglobin; platelet count; serum albumin; total bilirubin; alanine aminotransferase; aspartate aminotransferase; alkaline phosphatase; prothrombin time; blood urea nitrogen; serum creatinine; liver lesion aspirate and blood culture results; and in vitro bacterial susceptibility to antibiotics.

6. Radiological features of the abscess(es): size of the index abscess; number and location of the abscesses, if present; whether abscesses are unilocular or multilocular; the presence of gas in the abscess cavity; rim enhancement by contrast material; and the presence of the "cluster sign."

7. Drainage method employed: needle aspiration; continuous drainage using a percutaneous 8.5 French or 10 French pigtail catheter; or open surgical drainage.

8. Antimicrobial therapy and duration.

9. Outcome information: length of hospital stay; metastatic suppurative complications; and vital status at hospital discharge.

\section{Statistical analysis}

Continuous data were expressed as a mean with its associated standard deviation or range. Categorical data were expressed as the number of subjects with a specified condition, or a clinical variable and its corresponding percentage. Chi square, Student's $t$-test and Fisher's exact test were used when necessary. Logistic regression analysis was used to analyze the risk factors associated with KLA. A $P$-value $<0.05$ was considered statistically significant.

\section{Results}

Between January 1, 2007 and December 31, 2011, 49 patients were identified with PLA by the criteria listed in the Definitions section. Because of missing medical records, one patient was excluded. Twenty-nine patients $(60 \%)$ were male. The mean age of the cohort was 56 years (range: $20-83$ years). Thirty-one (65\%) patients were Caucasian, eleven (23\%) were Hispanic, five $(10 \%)$ were Asian, and one $(2 \%)$ was African-American. The most frequently associated conditions encountered were intra-abdominal infections in ten patients (20\%), diabetes mellitus in nine patients (18\%), and malignancy in nine patients (18\%). Diabetes mellitus was the only known risk factor for PLA in five patients. Seventeen patients (35\%) had no obvious cause or risk factor for PLA and were classified as having a cryptogenic liver abscess. The probable etiology of PLA in our cohort is illustrated in Table 1. Other associated comorbidities in the cohort included liver cirrhosis in three patients $(6 \%)$, congestive heart failure in two patients (4\%), and chronic kidney disease in one patient $(2 \%)$. The clinical features and biochemical parameters are summarized in Tables 2 and 3, respectively. In all of the reviewed cases, PLA diagnosis was established within 2 days of hospital admission. Furthermore, none of our cohort patients reported overseas travel.

\section{Radiological features}

Computed tomography was the most commonly used imaging modality for the diagnosis of PLA, as it was used in 
Table I Etiology of pyogenic liver abscess

Hepato-pancreatico-biliary; total of 20 cases (42\%)

- Benign

o Cholecystitis; four cases

o Cholelithiasis; four cases

o Liver cirrhosis; three cases

o Pancreatic abscess; one case

- Malignant

o Pancreatic cancer; five cases

o Hepatocellular carcinoma; one case

o Cholangiocarcinoma; one case

o Ampulla of Vater adenocarcinoma; one case

Extra-hepato-pancreatico-biliary; total of 6 cases (13\%)

- Benign

o Appendicitis; two cases

o Diverticulitis; one case

o Pelvic abscess; one case

o Infected omentum; one case

- Malignant

o Esophageal cancer; one case

Diabetes mellitus alone; five cases (10\%)

Cryptogenic liver abscess; 17 cases (35\%)

47 patients, with a sensitivity of $98 \%$ (46/47). One patient was diagnosed only by ultrasonography. Nineteen patients initially underwent sonographic examination of the liver, three of which did not detect the liver abscess ( $84 \%$ sensitivity). The radiological features are listed in Table 4.

\section{Microbiology}

Aerobic and anaerobic cultures of blood and abscesses were obtained from all patients. Abscess cultures were positive in $23(48 \%)$ patients; ten $(44 \%)$ were monomicrobial and 13 (56\%) were polymicrobial ( $\geq 2$ organisms isolated). Klebsiella spp. were the most commonly isolated bacteria (seven cases; 30\%); Klebsiella pneumoniae was found in five cases, and Klebsiella oxytoca in two cases. Viridans streptococci were the second most commonly isolated organisms, found in six $(26 \%)$ of the positive culture cases. Collectively, anaerobes (Fusobacterium sp., Bacteroid fragilis, Clostridium sp., and Prevotella sp.) were isolated in ten cases (43\%). Two specimens had Gram-positive staining but negative

Table 2 Clinical features of 48 patients with pyogenic liver abscess

\begin{tabular}{ll}
\hline Symptom/sign & Number (\%) \\
\hline Fever & $39(81 \%)$ \\
Abdominal pain & $35(73 \%)$ \\
Chills & $23(48 \%)$ \\
Gastrointestinal symptoms (nausea, vomiting, diarrhea) & $15(31 \%)$ \\
Septic shock & $3(6 \%)$ \\
Respiratory failure & $1(2 \%)$ \\
\hline
\end{tabular}

Table 3 Abnormal laboratory findings in pyogenic liver abscess patients

\begin{tabular}{ll}
\hline Laboratory test & Frequency (\%) \\
\hline Hypoalbuminemia $^{\mathrm{a}}$ & $45 / 46(98 \%)$ \\
Prolonged prothrombin time $^{\mathrm{b}}$ & $39 / 43(91 \%)$ \\
Leukocytosis $^{\mathrm{c}}$ & $38 / 48(79 \%)$ \\
Anemia $^{\mathrm{d}}$ & $36 / 48(75 \%)$ \\
Elevated alkaline phosphatase $^{\mathrm{e}}$ & $32 / 46(70 \%)$ \\
Elevated serum aspartate aminotransferase $^{\mathrm{f}}$ & $27 / 46(59 \%)$ \\
Elevated serum alanine aminotransferase $^{\mathrm{a}}$ & $24 / 46(52 \%)$ \\
Thrombocytosis $^{\mathrm{h}}$ & $16 / 48(33 \%)$ \\
Elevated blood urea nitrogen $^{\mathrm{i}}$ & $13 / 48(27 \%)$ \\
Elevated creatinine $^{\mathrm{j}}$ & $1 \mathrm{I} / 48(23 \%)$ \\
Hyperbilirubinemia $^{\mathrm{k}}$ & $7 / 45(16 \%)$ \\
\hline
\end{tabular}

Notes: aSerum albumin; normal range: $3.7-5.1 \mathrm{gm} / \mathrm{dL}$; ${ }^{b}$ prothrombin time; normal range: 10.7-13.5 seconds; 'peripheral white blood cell count; normal range: 4,000-10,600 cells $/ \mathrm{mm}^{3}$; dnormal hemoglobin range: $12-16 \mathrm{gm} / \mathrm{dL}$; enormal range: 38-126 U/L; 'serum aspartate aminotransferase; normal range: |5-4| U/L; sserum alanine aminotransferase; normal range: 14-54 U/L; ${ }^{h}$ normal range: $150,000-400,000$ cells $/ \mathrm{mm}^{3}$; 'blood urea nitrogen; normal range: $70-20 \mathrm{mg} / \mathrm{dL}$; inormal range: $0.5-1.4 \mathrm{mg} / \mathrm{dL}$; ${ }^{k}$ total bilirubin; normal range: $0.4-2.0 \mathrm{mg} / \mathrm{dL}$.

cultures; the first showed Gram-positive cocci in pairs, and the second showed Gram-positive cocci in pairs and Grampositive rods. Only five patients had confirmed bacteremia; three had concordant blood and abscess culture results, and two had bacteremia with negative cultures in the abscess. The organisms isolated from PLA patients are demonstrated in Figure 1.

Among the 14 isolated Gram-negative bacteria, four were third-generation cephalosporin-resistant microbes, among which three were extended spectrum beta-lactamase-producing bacteria. The same three bacteria were fluoroquinolone-resistant; there were no carbapenem-resistant isolates.

Patients with monomicrobial PLA were compared to patients with polymicrobial PLA in regards to age, sex, diabetes, chronic liver disease or cirrhosis, malignancy,

Table 4 Characteristics of liver abscesses on computed tomography

\begin{tabular}{ll}
\hline Computed tomography findings & $\begin{array}{l}\text { Number of } \\
\text { patients (\%) }\end{array}$ \\
\hline Number of abscess(es) & \\
Single & $26 / 48(54 \%)$ \\
Two or more & $22 / 48(46 \%)$ \\
Location & \\
Right lobe & $26 / 48(54 \%)$ \\
Left lobe & $12 / 48(25 \%)$ \\
Bilobar & $10 / 48(21 \%)$ \\
Unilocular:multilocular & $31: 13$ \\
Average size of the abscess (or of the largest & $6.3 \pm 2.7 \mathrm{~cm}$ in \\
abscess if there are multiple abscesses) & diameter \\
Rim enhancement & 20 \\
Gas in abscess cavity & 3 \\
Cluster sign & 9 \\
\hline
\end{tabular}




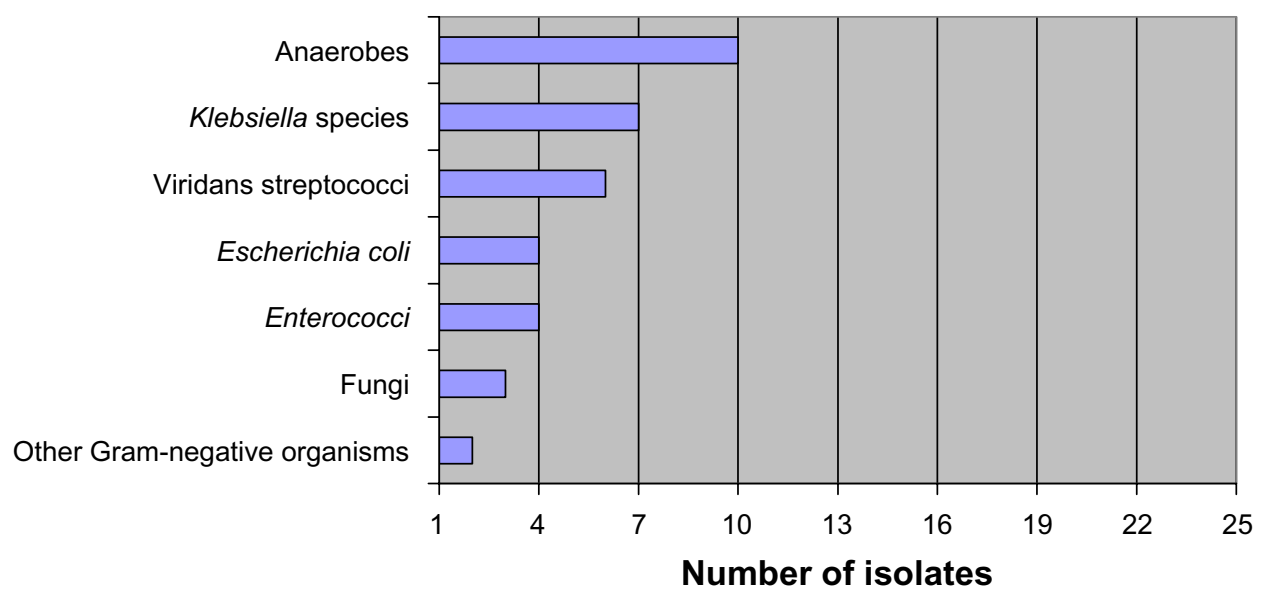

Figure I The number of organisms isolated from all positive liver abscess cultures $(n=23)$.

presentation including fever and white blood cell counts, and hospital length of stay. The difference between both groups was not statistically significant for any of these variables (data not shown).

\section{Klebsiella liver abscess}

All patients with KLA were Caucasian ( $n=7$ ) and five of them were male. The average age of this group was 66 years (range: 47-80 years). Three patients had pancreatic cancer, one patient had liver cirrhosis, and another patient was diabetic. Four patients had multiple abscesses, and the majority of cases had right hepatic lobe involvement (71\%). In the K. pneumoniae liver abscess group, two cases were monomicrobial and three cases were polymicrobial. Viridans streptococcal species were the additional bacterial species most frequently isolated in the polymicrobial KLA group, as it was isolated in three cases. The Klebsiella spp. isolated from the abscesses were resistant to ampicillin in all cases, but they were susceptible to all other tested antibiotics. All patients underwent successful percutaneous drainage. None of the patients had Klebsiella bacteremia or developed metastatic suppurative complications, although one patient had a positive blood culture for a viridans streptococcal species.

\section{Risk factors for Klebsiella liver abscess in the Caucasian group}

Caucasians with positive but non-KLA cultures (non-KLA; $\mathrm{n}=12)$ and those with negative abscess cultures $(\mathrm{n}=12)$ were grouped together for the statistical analysis. Comparisons were then made with Caucasians with KLA ( $n=7)$, and the results are found in Table 5. There was no mortality in either group. Age and the presence of malignancy were tested separately in a logistic regression model as risk factors for KLA. Neither of the independent variables reached statistical significance (age: $P=0.06$; malignancy: $P=0.06 ; R^{2}=0.082$ ). However, when age and the presence of malignancy were tested together in the same model (controlling for age), the model's $R^{2}$ value significantly improved $\left(R^{2}=0.19\right.$; odds ratio: $6.2 ; P=0.02$ ).

\section{Treatment and outcomes}

The best approach to drain a particular liver abscess was determined by the attending radiologist. When percutaneous aspiration was felt to be appropriate, typically a 20 -gauge, fine aspiration needle was used to drain the abscess cavity under radiological guidance. When continuous drainage was deemed necessary, a percutaneous 8.5 French or 10 French pigtail catheter was inserted. A follow up CT scan of the liver was typically performed 1-2 weeks later, and every 2 weeks thereafter if the abscess did not resolve on the first follow-up scan. Removal of the percutaneous drain was decided based on the patient's clinical and laboratory response.

Thirty-nine patients $(81 \%)$ underwent percutaneous drainage with an indwelling pigtail catheter. Six patients $(13 \%)$ underwent abscess aspiration via needle aspira-

Table 5 Clinical features of Caucasians with Klebsiella versus non-Klebsiella liver abscess

\begin{tabular}{llll}
\hline Clinical feature & $\begin{array}{l}\text { Klebsiella } \\
\text { liver abscess } \\
(\mathbf{n = 7})\end{array}$ & $\begin{array}{l}\text { Non-Klebsiella } \\
\text { liver abscess plus } \\
\text { negative abscess } \\
\text { cultures (n=24) }\end{array}$ & P-value \\
\hline Age; mean \pm SD & $66 \pm 12$ years & $62 \pm$ II years & 0.4 \\
Male:female (male \%) & $5: 2(71 \%)$ & 14:10 (58\%) & 0.5 \\
Diabetes; n (\%) & I (14\%) & $7(29 \%)$ & 0.4 \\
Cirrhosis; n (\%) & I (14\%) & $3(13 \%)$ & 0.4 \\
Cancer; n (\%) & $3(43 \%)$ & $5(21 \%)$ & 0.2 \\
\hline
\end{tabular}

Abbreviation: SD, standard deviation. 
tion only. The three remaining patients $(6 \%)$ were treated medically with intravenous antibiotics alone. One patient underwent open surgical drainage following a failed percutaneous drainage attempt.

All patients were started on antibiotics prior to abscess drainage. The time interval between antibiotic administration and drainage was less than 36 hours in all cases. Beta-lactam antibiotics were the most commonly used class, including 19 patients on imipenem, 14 on piperacillin/tazobactam, and three on meropenem. Six patients $(12.2 \%)$ received betalactam antibiotics alone; 33 patients $(67.3 \%)$ were treated with beta-lactam antibiotics plus some combination of vancomycin, metronidazole, and/or the antifungal agent fluconazole.

Fifteen patients $(30.6 \%)$ were discharged on the same empiric intravenous antibiotic regimen initiated on admission, including seven out of the 23 patients with positive culture results. Beta-lactam antibiotics continued to be the most commonly used medications at discharge, with meropenem, imipenem, and piperacillin/tazobactam being prescribed in 23 out of 33 patients.

The average duration of percutaneous drainage was $22 \pm 20$ days (range: $4-56$ days). The mean length of hospital stay was $20 \pm 22$ days (range: $1-82$ days). One patient in the study $(2 \%)$ died.

\section{Discussion}

In this retrospective study, the risk factors, microbiology, treatment, clinical course, and outcomes of 48 patients with PLA are reviewed and reported. In our series, we have been able to identify an underlying, predisposing disease process associated with the abscess formation in two-thirds of the cases including hepato-pancreatico-biliary system problems and cancer. Cancer was present in $29 \%$ of the patients with an identified problem, which supports recent reports suggesting that this is an emerging risk factor for PLA. ${ }^{3-5}$ Although it has been suggested that cryptogenic liver abscess is a sign of occult gastrointestinal malignancy, ${ }^{6}$ none of the patients with cryptogenic abscesses in this retrospective study had a screening colonoscopy performed during their index hospitalization.

Consistent with other studies, CT was more sensitive than ultrasonography in the detection of liver abscesses, with $98 \%$ being clearly identified by $\mathrm{CT} .^{7-9}$ The single false-negative CT examination in our series demonstrated diffuse mottled areas of low attenuation without a focal mass. A subsequent magnetic resonance imaging conducted in that patient showed a roundshaped mass in the liver. This abscess may have been missed by CT due to the lack of contrast enhancement, ${ }^{8}$ or perhaps due to an underlying diffuse liver abnormality that obscured it. ${ }^{9}$
An important finding in this study is that the incidence of KLA (30\% of all positive abscess cultures) was higher than expected, suggesting the possible emergence of Klebsiella spp. as an important cause of liver abscess in the United States. High rates of KLA have been seen in recent years in Asia, ${ }^{4,10}$ and they have also been reported in two studies in the United States. ${ }^{11,12}$ Malignancy was very common in the group with KLA in our study, indicating that cancer may be an important risk factor. Other than this, it is unclear why the incidence of KLA is increasing, although the expansion of the at-risk population could, at least partially, explain this phenomenon. $^{3}$

In Asia, diabetes has been found to be an important risk factor for KLA; up to $75 \%$ of patients with KLA in Asia have diabetes. ${ }^{4}$ While malignancy is known to impair the immune system, it has been rarely associated with KLA in the Asian population. In a retrospective Taiwanese study, ${ }^{13}$ malignancy was found in only $4 \%$ of KLA patients, and similar rates have been reported elsewhere. ${ }^{4,14}$ We and others in the United States have found a lower incidence of diabetes amongst KLA patients in particular, ranging between 15\%-33\% ${ }^{11,12}$ Despite literature reports from Asia where suppurative complications at distal sites have been seen in $12 \%-15 \%$ of KLA cases, ${ }^{4,14}$ we and others in the United States have not seen this. ${ }^{11,12}$

Overall, the mortality in our cohort is among the lowest reported; only one patient died from multiorgan system failure due to severe sepsis. The low mortality rate observed more specifically in the KLA patient subgroup of our cohort is in keeping with that of other recent reports from $\mathrm{Asia}^{3}$ and the United States. ${ }^{11,12}$ Our findings confirm that the percutaneous approach for liver abscess drainage is safe and effective when combined with appropriate antimicrobial therapy. ${ }^{15,16}$ It has been previously reported that the presence of malignancy is a poor prognostic factor in PLA patients. ${ }^{17}$ Although $19 \%$ of PLA patients in our cohort had cancer, all of them were discharged alive. We speculate that the low mortality observed in our cohort reflects the increasing awareness of the disease, early detection, and prompt initiation of appropriate drainage, along with the use of antimicrobial therapy.

The present study has some important limitations. First, it is a retrospective study, and some important and relevant information may not have been documented in the patient's medical chart by the staff members who cared for the patients. Second, strong conclusions cannot be made due to the small sample size. Third, patients were followed for a very short period of time after discharge; hence, long-term outcomes are not available. Fourth, culture samples of Klebsiella spp. were destroyed by the clinical microbiology laboratory a few days after the results 
were reported, according to usual policies and procedures. This precluded the reporting of detailed microbiological data on $K$. pneumoniae serotypes ( $\mathrm{K} 1$ and $\mathrm{K} 2$ ) and virulence factors like, rmpA and ma A. ${ }^{18}$ These data, admittedly, are considered a central tenet in the study of PLA due to K. pneumoniae.

In conclusion, PLAs continue to be an important, though uncommon, cause of morbidity among hospitalized patients. KLA in particular appears to be emerging as a cause of PLA in the United States. Although our patient sample size is small, it suggests that malignancy may be an important risk factor for KLA specifically, and this requires further investigation. Clinicians should maintain PLA in their differential diagnosis of patients who present with the risk factors and clinical scenarios of fever, abdominal pain, and liver lesions, since prompt diagnosis, percutaneous drainage of the abscess, and administration of intravenous antibiotics can shorten hospital stays and save lives.

\section{Acknowledgments}

We would like to thank Dr Richard Jordan for his continuous support. We thank Dr Robert Urban for his contributions. We would like to thank Patty Price, Jana Hutcherson, Sara Roos, and Megan Slaton for their help in the preparation of the manuscript.

\section{Author contributions}

Ahmad H Ali: principal writer of the manuscript; participated in the acquisition and analysis of the data. Roger D Smalligan: cowriter; participated in the data analysis and provided a critical revision of the manuscript. Mashrafi Ahmed: participated in the acquisition of the data and provided critical revision of the manuscript. Faisal A Khasawneh: cowriter; participated in the acquisition and analysis of the data; chief editor of the manuscript.

\section{Disclosure}

The authors report no conflicts of interest in this work.

\section{References}

1. Meddings L, Myers RP, Hubbard J, et al. A population-based study of pyogenic liver abscesses in the United States: incidence, mortality, and temporal trends. Am J Gastroenterol. 2010;105(1):117-124.
2. Alvarez Pérez JA, González JJ, Baldonedo RF, et al. Clinical course, treatment, and multivariate analysis of risk factors for pyogenic liver abscess. Am J Surg. 2001;181(2):177-186.

3. Tsai FC, Huang YT, Chang LY, Wang JT. Pyogenic liver abscess as endemic disease, Taiwan. Emerg Infect Dis. 2008;14(10): $1592-1600$.

4. Wang JH, Liu YC, Lee SS, et al. Primary liver abscess due to Klebsiella pneumoniae in Taiwan. Clin Infect Dis. 1998;26(6):1434-1438.

5. Greenstein AJ, Lowenthal D, Hammer GS, Schaffner F, Aufses AH. Continuing changing patterns of disease in pyogenic liver abscess: a study of 38 patients. Am J Gastroenterol. 1984;79(3):217-226.

6. Lai HC, Lin HC. Cryptogenic pyogenic liver abscess as a sign of colorectal cancer: a population-based 5-year follow-up study. Liver Int. 2010;30(9):1387-1393.

7. Halvorsen RA Jr, Foster WL Jr, Wilkinson RH Jr, Silverman PM, Thompson WM. Hepatic abscess: sensitivity of imaging tests and clinical findings. Gastrointest Radiol. 1988;13(2):135-141.

8. Halvorsen RA, Korobkin M, Foster WL, Silverman PM, Thompson WM. The variable CT appearance of hepatic abscesses. AJR Am J Roentgenol. 1984;142(5):941-946.

9. Quint LE, Glazer GM. CT evaluation of the bile ducts in patients with fatty liver. Radiology. 1984;153(3):755-756.

10. Chung DR, Lee SS, Lee HR, et al; Korean Study Group for Liver Abscess. Emerging invasive liver abscess caused by K1 serotype Klebsiella pneumoniae in Korea. J Infect. 2007;54(6):578-583.

11. Rahimian J, Wilson T, Oram V, Holzman RS. Pyogenic liver abscess: recent trends in etiology and mortality. Clin Infect Dis. 2004;39(11): 1654-1659.

12. Lederman ER, Crum NF. Pyogenic liver abscess with a focus on Klebsiella pneumoniae as a primary pathogen: an emerging disease with unique clinical characteristics. Am J Gastroenterol. 2005;100(2): 322-331.

13. Law ST, Kong Li MK. Is there any difference in pyogenic liver abscess caused by Streptococcus milleri and Klebsiella spp?: Retrospective analysis over a 10 -year period in a regional hospital. J Microbiol Immunol Infect. 2013;46(1):11-18.

14. Yang CC, Yen $\mathrm{CH}$, Ho MW, Wang JH. Comparison of pyogenic liver abscess caused by non-Klebsiella pneumoniae and Klebsiella pneumoniae. J Microbiol Immunol Infect. 2004;37(3):176-184.

15. Yu SC, Ho SS, Lau WY, et al. Treatment of pyogenic liver abscess: prospective randomized comparison of catheter drainage and needle aspiration. Hepatology. 2004;39(4):932-938.

16. Rajak CL, Gupta S, Jain S, Chawla Y, Gulati M, Suri S. Percutaneous treatment of liver abscesses: needle aspiration versus catheter drainage. AJR Am J Roentgenol. 1998;170(4):1035-1039.

17. Yeh TS, Jan YY, Jeng LB, et al. Pyogenic liver abscesses in patients with malignant disease: a report of 52 cases treated at a single institution. Arch Surg. 1998;133(3):242-245.

18. Yeh KM, Kurup A, Siu LK, et al. Capsular serotype K1 or K2, rather than magA and rmpA, is a major virulence determinant for Klebsiella pneumoniae liver abscess in Singapore and Taiwan. J Clin Microbiol. 2007;45(2):466-471.
International Journal of General Medicine

\section{Publish your work in this journal}

The International Journal of General Medicine is an international, peer-reviewed open-access journal that focuses on general and internal medicine, pathogenesis, epidemiology, diagnosis, monitoring and treatment protocols. The journal is characterized by the rapid reporting of reviews, original research and clinical studies across all disease areas.

\section{Dovepress}

A key focus is the elucidation of disease processes and management protocols resulting in improved outcomes for the patient.The manuscript management system is completely online and includes a very quick and fair peer-review system. Visit http://www.dovepress.com/ testimonials.php to read real quotes from published authors. 